\title{
¿Es efectivo que los antidepresivos aumentan el riesgo de suicidio?
}

\author{
Hernán Silva1, Juan Carlos Martínez². \\ Do antidepressants really \\ increase suicide rates in \\ childhood and adolescence?
}

The use of antidepressant in depressive illness results in a reduction of suicidal attempts and deaths due to suicide, conditions that are generally present in this disorder. Recently, the Federal Drug Administration (FDA) prohibited the use of antidepressants during childhood and adolescence. This decision was based on a supposed increase in suicidal thinking in these age groups. However, the evidence came from flawed clinical studies, some of them not even published, in which no significant differences were observed when compared to placebo. It is not possible to ascribe a direct responsibility to antidepressants, because depression, by definition, has suicidal ideation. On the contrary, the reduction of suicidal rates supports the effectiveness of these medications (Rev Méd Chile 2007; 135: 1195-201).

(Key words: Adolescent; Antidepressive agents; Child, preschool; Depression; Suicide)

Recibido el 5 de octubre, 2006. Aceptado el 27 de noviembre, 2006.

${ }^{1}$ Clínica Psiquiátrica Universitaria, Facultad de Medicina, Universidad de Chile. ${ }^{2}$ Servicio de Psiquiatría, Hospital Naval «Almirante Nef», Viña del Mar, Chile.

$\mathrm{E}^{\mathrm{n}}$ n junio del año 2003, el United Kingdom's Department of Health (UK) y la United States Food and Drug Administration (FDA) advirtieron públicamente sobre los cuidados con el uso de paroxetina en menores de 18 años. Seis meses más tarde, la British Medicines and Healthcare Products Regulatory Agency (MHRS) sugirió el cese del uso de antidepresivos en menores de 18 años, con excepción de fluoxetina. Esta sugerencia se basó, en parte, en tres estudios, dos de ellos no publicados, acerca del incremento de conductas suicidas en niños y adolescentes ${ }^{1}$.

Correspondencia a: Dr. Hernán Silva. Clínica Psiquiátrica Universitaria, Facultad de Medicina, Universidad de Chile. Av. La Paz 1003, Recoleta, Santiago, Chile. Teléfono: 9788237. Fax: 7776786. E mail: hsilva@med.uchile.cl
Estos estudios comparaban la prevalencia de ideación suicida de novo en pacientes tratados con paroxetina versus placebo, encontrando una incidencia de 3,7\% (14 en 378 pacientes) en el grupo tratado con paroxetina y 2,5\% (7 en 285 pacientes) en el grupo tratado con placebo, sin diferencias estadísticamente significativas $(p=0,5)^{2}$.

Paralelamente, en los Estados Unidos de Norteamérica, en una extensa revisión que incluyó 24 estudios, con un total de 4.100 pacientes (14 estudios en depresión, el resto en cuadros ansiosos y en déficit atencional hiperactivo), se encontró que los individuos medicados con antidepresivos tuvieron 1,8 veces más eventos suicidas en comparación con placebo $(3,8 \text { versus } 2,1 \%)^{3,4}$. Posteriormente, en marzo de 2004, la FDA sugirió restringir y vigilar el uso de antidepresivos en los pacientes adultos, ante la posibilidad de empeorar los sínto- 
mas depresivos y la ideación suicida, extendiendo la vigilancia a la fluoxetina, sertralina, paroxetina, fluvoxamina, citalopram, escitalopram, bupropion, venlafaxina, nefazodona y mirtazapina. En octubre del mismo año y tras evaluar 26 estudios, la FDA extendió la alerta al empleo de todos los antidepresivos (actuales y futuros), en menores de 18 años. Sin embargo, ninguno de los 26 estudios, tal como ocurrió en los reportes previos, evidenció algún caso de suicidio consumado y muchos de ellos no han sido dados a conocer a la comunidad científica, desconociéndose su rigurosidad metodológica y estadística ${ }^{5}$.

Por otra parte, la definición de suicidalidad empleada en los estudios señalados resulta imprecisa y extensa, incluyendo conductas como ideación suicida, autoagresiones (cortes) e intentos de autoeliminación.

Finalmente, en febrero de 2005, la alerta original fue cambiada y acotada por la FDA, precisando que el uso de inhibidores selectivos de la recaptación de serotonina (ISRS) produciría incremento de la ideación y de las conductas suicidas, no así del suicidio propiamente tal. En junio de ese mismo año, el mismo organismo americano advirtió nuevamente sobre la posibilidad de que los antidepresivos ISRS aumenten la suicidalidad en los adultos ${ }^{6}$.

\section{CRítica A LOS PLANTEAMIENTOS DE LA FDA}

A la luz de los antecedentes entregados, sse puede concluir que los antidepresivos provocan más suicidios que el placebo?, ¿cuál sería la frecuencia de las conductas suicidas en ese grupo de pacientes si no estuviesen recibiendo antidepresivos 0 placebo, es decir, observando el curso natural de la enfermedad, que de suyo tiene un alto porcentaje de mortalidad?

Son preguntas difíciles de responder, considerando la escasa información y evidencia al respecto. Sin embargo, antes de atribuir un poder suicidio-génico a los antidepresivos, debe tenerse en cuenta que los estudios en los que se basan los organismos reguladores norteamericanos y británicos no consideran importantes factores como la condición clínica previa y actual del paciente (falta absoluta de respuesta, respuesta parcial, remisión, recaída, etc), fracaso en la adherencia, discontinua- ción del tratamiento, uso de alcohol y drogas, grado de religiosidad del sujeto, parámetros farmacocinéticos (como una excesiva metabolización y un insuficiente nivel sérico del antidepresivo) y el tiempo transcurrido entre el inicio del tratamiento y la aparición de la ideación suicida o del acto autoagresivo ${ }^{7}$.

Al respecto, Jick y cols encontraron que, a medida que transcurre el tratamiento antidepresivo, disminuía el riesgo de suicidalidad. De ese modo, quienes llevaban entre 1 y 9 días de tratamiento, tenían 4 veces más probabilidad de ejecutar un acto suicida no fatal en comparación con quienes llevaban más de 90 días $^{8}$. Sería de interés conocer la metodología de los estudios señalados por la FDA, ya que el riesgo durante los primeros 9 días podría ser el mismo que para quienes no han iniciado la terapia farmacológica, atribuyéndose un acto suicida, sólo por un factor temporal, a un efecto directo de los antidepresivos.

Se cree que los antidepresivos tendrían un efecto protector contra el suicidio, mayor en las depresiones severas que en las leves. Al respecto, cuando son utilizadas para el diagnóstico de la enfermedad depresiva, las escalas estandarizadas de Hamilton (HDRS) y de Montgomery-Asberg (MADRS), difícilmente pueden diferenciar una depresión leve a moderada de un trastorno adaptativo. Por consiguiente, muchos estudios podrían considerar como depresivos a pacientes que no lo son, siendo estos falsos positivos» desorientadores a la hora de evaluar un medicamento. De hecho, la eficacia del placebo en el tratamiento de la depresión es elevada, entre otras razones, por la inclusión de trastornos de adaptación en los estudios. En estos casos, la mejoría del paciente se basa más bien en el manejo interpersonal o psicoterapéutico que permite una resolución 0 una adaptación a las situaciones que originaron el trastorno.

Cuando la incapacidad de adaptación es secundaria a rasgos anormales de personalidad, y si además se asocia a un ambiente disfuncional, las estrategias utilizadas para hacer frente a los conflictos son escasas y se despliegan de modo rígido. En estos casos, un acto suicida puede ser un modo primitivo de modificar las condiciones del entorno, pudiendo ser particularmente elevada su prevalencia, con un trasfondo mayoritariamente impulsivo, ganancial, inauténtico y sin 
intención real de muerte. Un intento suicida o una autoagresión en un paciente que sadopta»y muestra»su rol de enfermo, podría injustamente atribuirse al efecto directo de un antidepresivo.

Resulta dudoso atribuir al antidepresivo un aumento en las conductas suicidas, en la medida en que la ideación suicida y los actos que potencialmente le acompañan son condiciones inherentes a los trastornos del ánimo. El 60\%-70\% de los pacientes que presentan un cuadro depresivo experimenta ideación suicida, y entre $10 \%$ y $15 \%$ de los pacientes depresivos comete suicidio ${ }^{9}$. A la luz de estas cifras, es necesario aclarar que los estudios presentados por la FDA sólo consideran el número de pacientes que incrementan la suicidalidad (pensamientos, autoagresiones, actos suicidas), sin hacer referencia al número de pacientes que disminuyen la ideación o los intentos autolesivos con el tratamiento antidepresivo.

En los estudios presentados, la suicidalidad se incrementaba en no más de $2 \%$ sobre el placebo ${ }^{2-}$ 4. Si recordamos que $60 \%$ a $70 \%$ de los depresivos presenta ideación suicida (sin incluir autoagresiones ni intentos), el incremento de $2 \%$ no tendría ningún impacto clínico, versus las ventajas del antidepresivo sobre una población de alto riesgo. En general, se considera que un fármaco produce un efecto secundario significativo cuando éste se presenta en al menos $10 \%$ de los sujetos que lo reciben, o cuando tiene una diferencia de $4 \%$ por sobre el placebo, situación que no se produce en los casos de la suicidalidad por antidepresivos.

$\mathrm{Si}$ se hubiesen obtenido diferencias significativas con el placebo, se podría pensar que los antidepresivos realmente inducen suicidalidad. Sin embargo, esos hallazgos también podrían ser cuestionados, ya que es dudoso que los investigadores, por razones éticas, hayan asignado placebo $\mathrm{y}$ antidepresivos a pacientes con igual severidad depresiva. En tal caso los grupos no serían comparables.

Una exacerbación de la suicidalidad podría deberse a una mayor gravedad del cuadro clínico $y$, en tal caso, es posible que una monoterapia 0 el fármaco utilizado sean insuficientes para controlar la intensidad de dichos síntomas.

En ocasiones, un fármaco útil para la mayoría de los pacientes resulta problemático»en otros. La resistencia a los agentes antimicrobianos es un fenómeno que complica el uso clínico de dichos agentes. Del mismo modo, la provocación de nuevas arritmias o el incremento en frecuencia de una arritmia persistente ocurre con todos los agentes antiarrítmicos en $6 \%$ a $25 \%$ de los casos, lo que recibe el nombre de efecto proarritmogénico», observándose en pacientes con una isquemia cardiaca silente ${ }^{10}$. En este sentido, un factor que puede provocar un eventual incremento de la suicidalidad es el viraje de depresión a manía, particularmente a manía disfórica o irritable, en pacientes bipolares tratados con antidepresivos sin estabilizadores del ánimo asociados. Muchos pacientes depresivos, aparentemente monopolares, son bipolares en los que el polo maníaco o hipomaníaco no se ha manifestado o ha pasado desapercibido. Pese al riesgo de viraje a manía, menos de la mitad de los bipolares que usan antidepresivos, lo hace en asociación a un estabilizador ${ }^{11}$. Por otra parte, los bipolares no diagnosticados tienen 4 veces más riesgo de intentar suicidio y $50 \%$ más probabilidad de ser hospitalizados respecto a quienes no tienen una enfermedad bipolar ${ }^{11}$. La FDA no ha considerado que las consultas por episodios depresivos, tanto monopolares como bipolares, han aumentado notablemente durante la última década y con ello la prescripción de antidepresivos, por lo que la exposición a dichos fármacos resulta evidentemente mayor.

Mientras más grave es una depresión, es más probable que el paciente consulte al médico y que reciba tratamiento con uno o más antidepresivos, eligiéndose, por lo general, los fármacos más modernos, que son más seguros. En estos casos, la severidad de la depresión establece una relación entre quienes usan antidepresivos y el mayor riesgo suicida, que es atribuible a la intensidad del cuadro clínico. Al respecto, Mines y colaboradores (2005) reportan que la venlafaxina, uno de los antidepresivos más cuestionados, es prescrita a un grupo de pacientes depresivos particularmente graves. Por ejemplo, en comparación con los pacientes que habían sido tratados con fluoxetina y citalopram, los que recibían venlafaxina tenían 2,7 y 2,4 veces más antecedentes de un mayor número de conductas suicidas previas; 2,7 y 2,8 veces más hospitalizaciones por depresión y 6,1 y 4,3 veces más hospitalizaciones por intentos de suicidio. Igualmente, quienes estaban en tratamiento con venlafaxina, tenían 6,9 y 4,0 más 
probabilidad de haber recibido uno o más antidepresivos en comparación con quienes recibían fluoxetina y citalopram y 6,5 y 3,1 veces mayor probabilidad de haber recibido 2 o más antidepresivos durante el último año ${ }^{12}$. Lo anterior confirma la hipótesis de que los fármacos catalogados como suicido-génicos son asignados a los grupos de mayor riesgo.

En psiquiatría infanto-juvenil, los niños y adolescentes, generalmente, no acuden al psicólogo o al psiquiatra por sí mismos, sino que son llevados por sus padres o por un adulto cercano. Habitualmente lo que motiva la consulta no son los síntomas afectivos, sino más bien algunas condiciones asociadas a la depresión como molestias físicas inespecíficas (dolor abdominal, cefaleas, etc), humor irritable (que lleva a conductas oposicionistas-desafiantes), disminución del rendimiento escolar, rechazo escolar, alteraciones del sueño, del apetito, etc ${ }^{13}$. Esto hace que la consulta sea tardía y cuando los padres deciden consultar al psiquiatra, probablemente la enfermedad lleve bastante tiempo de evolución, o presente una mayor severidad. Por lo demás, los síntomas conductuales favorecen el error diagnóstico, no sólo en quienes carecen de pericia en temas relacionados con salud mental, sino incluso entre los especialistas; además la comorbilidad con otros diagnósticos psiquiátricos alcanza a 88\%, situación que complica aún más la clínica, evolución y tratamiento ${ }^{13}$.

Creemos que antes de preocuparse o de temer el empleo de un antidepresivo, el clínico deberá considerar el rol de los factores psicosociales y del medio ambiente, así como algunos eventos específicos al evaluar un riesgo suicida. Por ejemplo, una pérdida reciente, un pobre soporte social, una ruptura amorosa, un evento biográfico humillante, pueden afectar la letalidad de un intento suicida ${ }^{14}$. La observación anterior cobra valor si consideramos que dos tercios de los adultos suicidas consultaron al psiquiatra el mes previo a su muerte. En adolescentes, la situación puede ser más compleja, al ser un grupo más impulsivo y al estar día a día bajo el escrutinio y la crítica de padres y pares ${ }^{15}$.

Quizás la impulsividad señalada explique que $37 \%$ de los adolescentes no evidenciaron cambios importantes en las horas previas al intento suici$\mathrm{da}^{15}$. Sin embargo, Blumenthal (1988), tras un seguimiento de 10 años, encontró que $91 \%$ de los eventos suicidas podría haberse prevenido si se hubiese puesto atención al reporte de la «desesperanza» ${ }^{6}$. Por lo tanto, una entrevista orientada a la detección de éste y de otros síntomas, resulta importante en la prevención del suicidio. Al respecto, y aludiendo al papel del litio en la reducción del riesgo suicida, algunos autores sugieren que, entre otras causas desconocidas, los controles plasmáticos periódicos implican un contacto más cercano entre el médico y el paciente, lo cual ejercería un rol contenedor frente a la ideación autoagresiva ${ }^{17}$.

\section{EVIDENCIA A FAVOR DE LOS ANTIDEPRESIVOS}

A pesar del punto de vista de la FDA, existe fuerte evidencia de que el empleo de los antidepresivos se ha asociado con reducción del riesgo suicida. Desde los inicios de la terapéutica en psiquiatría, hay una progresiva y significativa disminución en las tasas de suicidio entre los pacientes depresivos. Así, en la "era preterapéutica" (1900-1939) dichas tasas eran de 6,3 casos por cada 1.000 pacientes; en la "era de la terapia electroconvulsiva" (1940-1959) se redujeron a 5,7 por 1.000 pacientes y en la "era de los antidepresivos" (1960-1992) a 3,3 por 1.000 pacientes $^{18}$. Del mismo modo, desde 1985 a 1999 la tasa de suicidios en los Estados Unidos de Norteamérica disminuyó de 12,4 por 100.000 habitantes a 10,7 por 100.000 (reducción de 13,5\%), mientras la prescripción de los antidepresivos (fundamentalmente ISRS), se incrementó cuatro veces. La declinación fue más pronunciada en las mujeres, coincidente con que éstas recibieron dos veces más antidepresivos que los hombres ${ }^{19}$. Por su parte, en los adolescentes, el aumento al doble en el uso de antidepresivos se asoció a una disminución de 0,23 suicidios cada 100.000 adolescentes por año ${ }^{20}$.

Diversos estudios indican que sólo una pequeña cantidad de suicidas estaba en tratamiento al momento de su muerte ${ }^{21}$. De acuerdo a Gray y colaboradores (2002) sólo $1 \%$ de los suicidas jóvenes estaba en tratamiento en el sistema público de salud, y sólo en 3\% fue posible detectar psicotrópicos en el plasma durante la autopsia ${ }^{22}$. Otros estudios toxicológicos revelan que un muy 
bajo porcentaje de los pacientes que recibían antidepresivos, tenía niveles plasmáticos detectables con posterioridad a su muerte, lo que permite concluir que el suicidio ocurría en quienes no respetaban las indicaciones o en quienes habían suspendido el tratamiento. De hecho, sólo $8 \%$ a $20 \%$ tendría un examen positivo para antidepresivos en sangre al momento de su muerte. Es decir, la mayoría de los pacientes que comete suicidio no habría tomado su medicación, por lo menos el día o los días previos al suicidio ${ }^{23-26}$.

Como los trastornos depresivos están presentes en $50 \%$ a $90 \%$ de los casos de suicidio $27-29$, y la falta de adherencia a los ISRS en la población general fluctúa entre $15 \%$ y $20 \% 30$, una buena relación y observación por parte del médico tratante, padres y familiares, así como la contención medio-ambiental, podrían ser factores protectores de suicidio. Pese a ello, la falta en la respuesta para un ISRS es de alrededor de $20 \%$ a $30 \% 31$, debiendo considerarse el ajuste de la dosis 0 el cambio de la medicación.

El predictor más potente de un futuro intento suicida es el antecedente de un intento previo. Más de dos tercios de quienes han intentado la autoeliminación repetirán dicha conducta. Más aún, cada intento suicida aumenta el riesgo de recurrir a métodos más agresivos y eficaces ${ }^{7}$. Quienes intentan suicidarse, tendrían 9 veces más probabilidad de tener conductas autolesivas, como ingesta de una sobredosis de fármacos y automutilaciones. Por su parte, quienes finalmente cometen suicidio, habrían tenido 15 veces más conductas autoagresivas en relación a los controles normales 32 .

La acatisia (sensación de inquietud, impaciencia, intranquilidad, ansiedad, insomnio o incomodidad generalizados) está fuertemente asociada a abandono precoz de tratamiento. La acatisia secundaria a los antidepresivos, a menudo se presenta durante los primeros 10 a 14 días de tratamiento, siendo comúnmente ignorada o confundida con ansiedad o con empeoramiento de la condición original ${ }^{33}$. Tendría una incidencia que varía entre $9,8 \%$ y $25 \%{ }^{34}$. Su influencia en el suicidio podría deberse a que reduce la adherencia al tratamiento antidepresivo.
Con el empleo de la primera generación de antidepresivos, tricíclicos e inhibidores de la monoaminooxidasa (IMAOS) clásicos, se observó que el período de mayor niesgo suicida ocume al inicio del tratamiento, cuando el paciente comienza a sentirse mejor, porque el efecto activador de los antidepresivos aparece antes que mejore el estado de ánimo. El paciente con ideación, o determinación suicida, encuentra en ese momento la energía necesaria para llevar adelante su propósito, energía de la que carecía en el momento más profundo de la depresión. Asimismo, las frecuentes fluctuaciones del ánimo que preceden a una mejonía estable, pueden llevar a algunos pacientes a caer en estados transitorios de depresión profunda, los que pueden resultar insoportables por contraste con el bienestar recientemente experimentado. Por esta razón es necesario advertir al paciente de estas fluctuaciones y asegurarle que no significan un fracaso del tratamiento, idea que puede llevarlos a la desesperanza y al suicidio.

\section{CONCLUSIONES}

La atribución de un rol suicido-génico a los antidepresivos resulta contradictoria con los reportes de la literatura. Para llegar a una conclusión de esa naturaleza, habría que depurar todos los factores biológicos, psicológicos y sociales, que impactan en concretar un acto suicida, ya que se trata de un hecho multifactorial, no atribuible a una causa aislada o específica.

Los estudios en que se basan los organismos reguladores norteamericanos y británicos para advertir el riesgo de inducción de conductas suicidas con el empleo de antidepresivos, carecen de una metodología rigurosa y sus resultados no alcanzan significación estadística. Quienes trabajan a diario en salud mental y particularmente quienes tratan pacientes con trastornos del ánimo, deben mantener una actitud crítica frente a dichas recomendaciones, en la medida en que no se fundamentan en evidencias sólidas. Enfrentados a una patología de elevada frecuencia y de tan alto costo personal y social, puede constituir un riesgo mayor abstenerse de emplear fármacos de eficacia demostrada, como los antidepresivos. 


\section{REFERENCIAS}

1. MÖLER HJ. SSRIs: Are the Accusations Justified? World J Biol Psychiatry 2004; 5: 174-5.

2. Keuer MB, Ryan ND, Strober M, Kiein RG, Kutcher SP, Birmaher B ET AL. Efficacy of Paroxetine in the Treatment of Adolescent Major Depression: A Randomized, Controlled Trial. J Am Acad Child Adolesc Psychiatry 2001; 40: 762-72.

3. BRent DA, BIRMAHER B. British warnings on SSRIs questioned. J Am Acad Child Adolesc Psychiatry 2004; 43: 4.

4. Goode E. British waming on antidepressant use for youth. The New York Times. December 11, 2003. p. A1.

5. Cheung AH, Emsle GJ, Mayes TL. Review of the efficacy and safety of antidepressants in youth depression. J Child Psychol Psychiat 2005; 46: 735-54.

6. Food and Drug Administration. Suicidality in adults being treated with antidepressant medications. FDA Public Health Advisory, June 30, 2005. Disponible en: http://www.fda.gov/cder/drug/ advisory/SSRI200507.htm. Acceso 11 de julio, 2005.

7. RiHMeR Z, AKISKaL H. Do antidepressants t(h)reat(en) depressives? Toward a clinically judicious formulation of the antidepressant-suicidality FDA advisory in light of declining national suicide statistics from many countries. J Affect Disord 2006; 94: 3-13.

8. Jick H, KaYe JA, Jick SS. Antidepressants and the risk of suicidal behaviors. JAMA 2004; 292: 33843.

9. MoLer HJ. Suicide, suicidality and suicide prevention in affective disorders. Acta Psychiatr Scand 2003; Suppl. 418: 73-80.

10. PodRID PJ. Proarrhythmia, a serious complication of antiarrhythmic drugs. Curr Cardiol Rep 1999; 1: 289-96.

11. Shia L, Thiebaudi P, McCombsb JS. The impact of unrecognized bipolar disorders for patients treated for depression with antidepressants in the fee-for-services California Medicaid (Medi-Cal) program. J Affec Disord 2004; 82: 373-83.

12. Mines D, HiL D, Yu H, Novew L. Prevalence of risk factors for suicide in patients prescribed venlafaxine, fluoxetine, and citalopram. Pharmacoepidemiol Drug Saf 2005; 14: 367-72.
13. Emsle GJ, Mayes TL. Depression in Children and Adolescents: Guide to Diagnosis and Treatment. CNS Drugs 1999; 11: 181-9.

14. Grupp-Phelan J. The suicidal pediatric patient: an emergency medicine focus. Clin Ped Emerg Med 2003; 4: 141-7.

15. KienhorsR IC, De Wilde EJ, Diekstra RF. Adolescents image of their suicide attempt. J Am Acad Child Psychiatry 1995; 34: 623-8.

16. Blumenthal SJ. Suicide: A guide to risk factors, assessment, and treatment of suicidal patients. Med Clin North Am 1988; 72: 937-71.

17. Tondo L, Ghiani C, Albert M. Pharmacologic interventions in suicide prevention. J Clin Psychiatry 2001; 62 (suppl 25): 51-55.

18. O’Lfary D, Pay Kel E, Todd C, Vardulaki K. Suicide in primary affective disorders revisited: a systematic review by treatment era. J Clin Psychiatry 2001; 62: 804-11.

19. Grunebaum MF, Eus SP, L S, Oquendo MA, Mann $\mathrm{JJ}$. Antidepressants and suicide risk in the United States, 1985-1999. J Clin Psychiatry 2004; 65: 1456-62.

20. Olfson M, Shaffer D, Marcus SC, Greenberg T. Relationship between antidepressant medication treatment and suicide in adolescents. Arch Gen Psychiatry 2003; 60: 978-82.

21. Shaffer D, Gould M, Fisher P, Trautman P, Moreau D, Kifinman M et al. Psychiatric diagnosis in child and adolescent suicide. Arch Gen Psychiatry 1996; 53: 339-48.

22. Gray D, AchiLes J, KeIIER T. Utah youth suicide study, phase I: Government agency contact before death. J Am Acad Child Adolesc Psychiatry 2002; 41: 427-43.

23. Isacsson G, Bergman U, Rich CL. Antidepressants, depression and suicide: an analysis of the San Diego study. J Affect Disord 1994; 3: 277-86.

24. Isacsson G, Holmgren P, Druid H, Bergman U. The utilization of antidepressants - a key issue in the prevention of suicide: an analysis of 5281 suicides in Sweden during the period 1992-1994. Acta Psychiatr Scand 1997; 96: 94-100.

25. Henriksson S, Boethius G, Isacsson G. Suicides are seldom prescribed antidepressants: findings from a prospective prescription database in Jamtland county, Sweden, 1985-95. Acta Psychiatr Scand 2001; 103: 301-6.

26. Marzuk PM, Tardiff K, Leon AC, Hirsch CS, Stajic M, Hartwell N, Portera L. Use of prescription psycho- 
tropic drugs among suicide victims in New York City. Am J Psychiatry 1995; 152: 1520-2.

27. Apter A, Bieich A, King RA, Kron S, Fluch A, Jotier $\mathrm{M}$, CoHEN DJ. Death without warning? Arch Gen Psychiatry 1993; 30: 138-42.

28. Marttunen MJ, Aro HM, Henriksson MM, LönNovist JK. Mental disorder in adolescent suicide. Arch Gen Psychiatry 1991; 48: 834-9.

29. Cheng AT. Mental illness and suicide. Arch Gen Psychiatry 1995; 52: 594-603.

30. Schrader E, Meier B, Brattström A. Hypericum treatment of mild-moderate depression in a placebo-controlled study. Human Psychopharmacol Clin Exp 1998; 3: 163-9.
31. Zonda T. Depression and Suicidal Behavior. Crisis 2005; 26: 34-5.

32. Martínez C, Rietbrock S, Wise L, Ashby D, Chick J, Moseley J ET AL. Antidepressant treatment and the risk of fatal and non-fatal self harm in first episode depression: nested case-control study. BMJ 2005; 330: 389.

33. LANE RM. SSRI-induced extrapyramidal sideeffects and akathisia: implications for treatment. J Psychopharmacol 1998; 12: 192-214.

34. HANSEN L. A critical review of akathisia, and possible association with suicidal behavior. Hum Psychopharmacol Clin Exp 2001; 16: 495-505. 